\title{
The Importance of Duration in the Prognosis of Facial Nerve Palsy and Its Treatment-A Case Report
}

\section{Nayak $\mathrm{PS}^{1}$, Debnath $\mathrm{P}^{2 *}$, Harugop AKS ${ }^{3}$ and Patil $\mathrm{PH}^{4}$}

${ }^{1}$ Assistant Professor, Department of ENT and HN surgery, KAHER'S Jawaharlal Nehru Medical College, India

${ }^{2}$ Post Graduate, Department of ENT and HN surgery, KAHER'S Jawaharlal Nehru Medical College, India

${ }^{3}$ Head of department and Professor, Department of ENT and HN surgery, KAHER'S Jawaharlal Nehru Medical College, India

${ }^{4}$ Professor, Department of ENT and HN surgery, KAHER'S Jawaharlal Nehru Medical College, India

*Corresponding author: Paramita Debnath, Post Graduate, Department of ENT and HN surgery, KAHER'S Jawaharlal Nehru Medical College, Belagavi, Karnataka 590010, India, Email: Paramitadebnath74@gmail.com

\section{Abstract}

Background: Facial nerve palsy is considered to be one of the most common consequence of untreated chronic suppurative otitis media. The incidence has significantly reduced over the decade due to better imaging technology and use of early antibiotics and better surgical procedures but if left untreated, it has a significant impact over the patient's day to day life and markedly affects patient's social life and causes serious psychological damage. An appropriate and prompt management ensures healing of the palsy without sequelae.

Case Report: We report a case of bilateral chronic suppurative otitis media with unilateral right grade 4 facial nerve palsy which happened within a duration of 1 month for which patient underwent right modified radical mastoidectomy following which she had steady improvement.

Conclusion: This case highlights that in patients with middle-ear cleft cholesteatoma the surgical approaches vary according to duration of palsy and post-operative improvement in facial nerve function can be anticipated in cases of acute palsy. Surgical interventions have been proved to have a good effect in restoration of facial nerve palsy in cases of shorter duration as compared to those of sudden onset and longer duration.

Keywords: Facial Nerve Palsy; Cholesteatoma; Radical mastoidectomy

\section{Introduction}

Facial nerve palsy is considered to be the one of the most common complications following middle ear infections. The unique anatomy the nerve makes it more vulnerable for palsy as compared to other cranial nerves. Among all other cause's cholesteatoma is considered to be the leading cause of the facial nerve palsy and tympanic segment is most commonly affected. It has a significant impact over the patient's day to day life and affects the patient both physically as well as emotionally, though it is not a life-threatening complication. Prompt diagnosis followed by early surgical intervention plays the key role in the treatment. We report a case of bilateral chronic suppurative otitis media with unilateral right grade 4 
facial nerve palsy for which patient underwent right modified radical mastoidectomy via a transmastoid approach which revealed the presence of labyrinthine fistula and dehiscence of sigmoid sinus with facial nerve decompression. Following which along with facial physiotherapy patient showed gradual improvement.

\section{Case Report}

A 20-year-old female presented to us with complaints of throbbing type of right ear pain for 1 month followed by which she developed deviation of angle of mouth to left which was sudden in onset, progressive, did not relieve on taking medications associated with drooling of saliva. She has developed right ear discharge for 1 year which aggravated since past 15 days, scanty in amount, greenish, purulent, foul-smelling, continuous, not relieved on taking medications.

ENT examination revealed right ear polyp arising from posterior wall of external auditory canal. On probing, firm in consistency, bleeds on touch non-tender, tympanic membrane not visualized. Left ear examination revealed large central perforation. Facial nerve examination showed right grade 4 facial nerve palsy with bells sign positive. Rest of ENT examination was normal Figure 1.

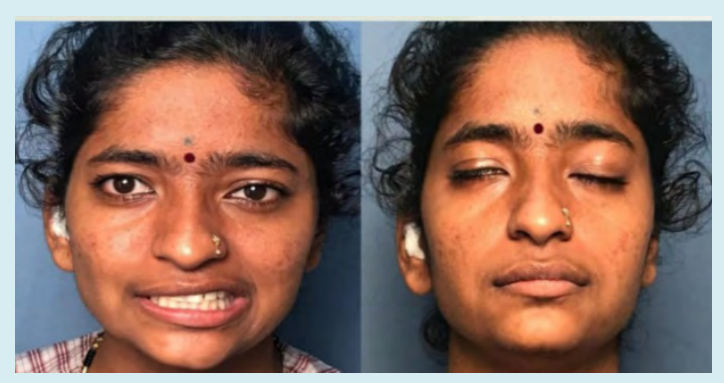

Figure 1: Pre-operative images showing right grade 4 facial nerve palsy with bells sign positive.

She was started on iv antibiotics, iv steroid therapy and analgesics. Pure tone audiometry revealed bilateral severe conductive hearing loss. Xray mastoid Schullers view revealed radiolucent cavity in right ear.

Following which patient underwent right canal wall down mastoidectomy with facial nerve decompression by transmastoid route. Intra operatively cholesteatoma was found involving the attic, aditus, antrum, eroding the mastoid cortex and extending till the eustachian tube, compressing the distal 1/3rd of horizontal and vertical segment of facial nerve. Dehiscence of sigmoid sinus was noted, labyrinthine fistula was seen in dome of lateral semicircular canal, membranous labyrinth was intact. Cholesteatoma was meticulously removed Figure 2. Harvested Temporalis fascia graft was placed over facial canal, lateral semi-circular canal and middle ear was obliterated with soft tissue and adequate meatoplasty was done. Post operatively patient was followed up every week till 4 weeks along with facial physiotherapy, facial nerve showed steady improvement with return to normal function at the end of $4^{\text {th }}$ week (Figure 3).
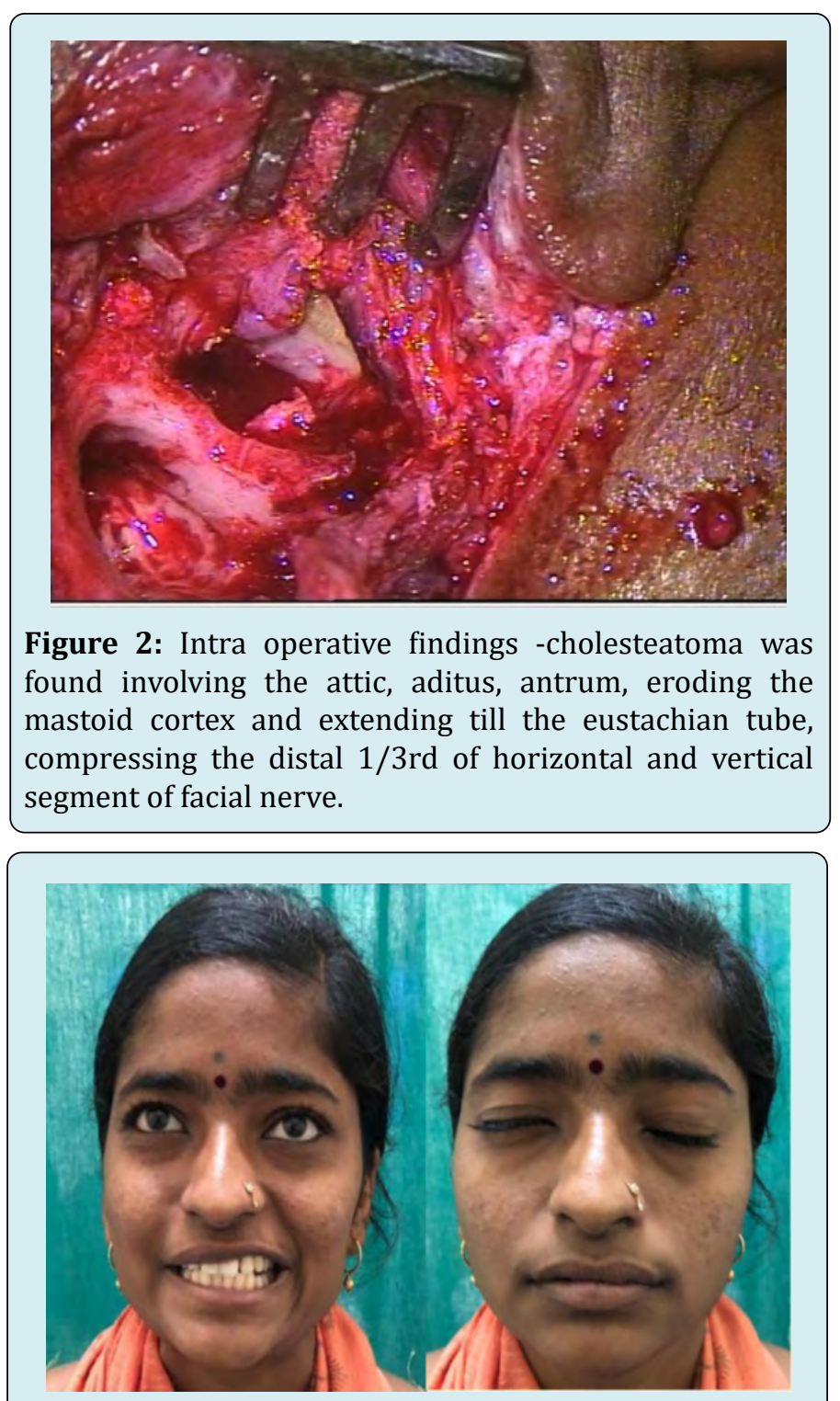

Figure 3: Post-operative findings-facial nerve showed steady improvement with return to normal function at the end of $4^{\text {th }}$ week.

\section{Discussion}

Facial nerve is considered as one of the most unique cranial nerve, it is a mixed nerve and is more vulnerable to injury owing to its course through the narrow and tortuous 


\section{Otolaryngology Open Access Journal}

fallopian canal. In human being's facial expressions forms an important mode of communication [1]. Facial nerve palsy has various etiological factors that includes infectious, neurologic, congenital, neoplastic, traumatic, systemic, and iatrogenic causes. Middle ear infections remain the most common cause responsible for facial nerve palsy. According to studies, $3.1 \%$ of all cases of facial palsy is seen due to chronic suppurative otitis media with cholesteatoma. The palsy can be either acute or insidious in nature. The common causes are acute suppurative otitis media (ASOM), ASOM with Mastoid abscess, and CSOM with cholesteatoma, temporal bone injury and fracture. The prognosis following palsy depends upon various factors including the duration and mode of onset, etiological factors [2]. The incidence of facial paralysis in relation to cholesteatoma is around 0.04$0.16 \%$ [3]. This figure is decreasing ever since the use of diagnostic tools like HRCT temporal bone and the invention of micro ear surgery.

The pathophysiology leading to otitic facial nerve palsy in chronic suppurative otitis media includes osteitis, bone erosion, external compression, oedema and inflammation of the nerve [4]. Facial palsy can occur secondary to ASOM and acute mastoiditis, is usually due to severe infection affecting the facial nerve in the presence of congenital dehiscence of the fallopian canal, usually in its tympanic segment and is commonly seen in children. The palsy usually responds well to antibiotics and myringotomy with a complete recovery. Rarely, a surgical decompression may be required in the presence of a coalescent mastoiditis. In contrast, facial palsy is 3-4 times more common in cholesteatoma than in ASOM and is due to the erosion of the fallopian canal by the cholesteatoma matrix. Involvement of the facial nerve is directly proportional to the size and extent of the cholesteatoma, which determines the intensity of infection, which in turn is responsible for the palsy. The treatment modality of choice in such cases is surgical decompression. The major difference lies in the fact that cholesteatoma cause a slower onset type of palsy while acute infections like mastoiditis superimposed with cholesteatoma causes a rather faster onset. Facial palsy tends to occur early in tuberculous otitis media due to erosion of bone by the granulation tissue and formation of bony sequestra.

Palsy following such causes has a great impact on the patient's life both socially and emotionally. Diagnosing the palsy is based on proper clinical examination followed by electrophysiological testing while CT scan provides a definitive diagnosis and the staging of paralysis also plays a key role. As reported by Ikeda, et al. in their study conducted in 2005 duration of onset of facial paralysis is a critical factor, in the surgical timing [5]. The management of facial paralysis requires a more complex reconstructive procedure with lot of functional and cosmetic deficits involved in the palsy [6].
In acute cases facial nerve decompression and facial nerve repair remains the modality of choice while chronic cases require regional or free muscle transfer.

In our case the patient gives history of long-standing ear discharge. The patient failed to do regular follow ups which lead to acute onset of facial nerve palsy for which she underwent modified radical mastoidectomy along with trans mastoid facial nerve decompression. She showed a steady recovery following the surgery. According to a study conducted by Kumar, et al. facial nerve decompression performed in palsy cases of longer duration has poorer prognosis and recovery of muscle power may not be complete [7].

\section{Conclusion}

Thus, to conclude for middle-ear cleft cholesteatoma the surgical approaches vary according to duration of palsy and post-operative improvement in facial nerve function can be anticipated in cases of acute palsy while regular follows ups and early diagnosis using top diagnostic tests remain the key in diagnosis.

\section{Clinical Significance}

In cases of facial nerve palsy-the duration of palsy remains the most important prognostic factor in the management plan. In a developing country like ours where most of the population cannot afford CT scan the diagnosis hugely depends upon clinical examination and Xray mastoids. Modified radical mastoidectomy with facial nerve decompression can be tried in such cases with acute onset of palsy as the post-operative results are high yielding and caries a better prognosis.

\section{References}

1. Sreekanth G, Sravan Kumar CH, Ahmed N, Kumar P (2016) A Study of Etiopathology and Management of Facial Palsy at a Tertiary Care Institute. Journal of Dental and Medical Sciences 15(7): 71-75.

2. Swamy DR, Kumar BR (2018) A Clinical Analysis of Facial Nerve Paralysis due to Inflammatory Diseases of the Middle Ear and the Role of Early Decompression. Int J Sci Stud 6(8): 115-119.

3. Siddiq MA, Hanu Cernat LM, Irving RM (2007) Facial palsy secondary to cholesteatoma: Analysis of outcome following surgery. J Laryngol Otol 121(2): 114-117.

4. Kim J, Jung GH, Park SY, Lee WS (2012) Facial Nerve Paralysis due to Chronic Otitis Media: Prognosis in Restoration of Facial Function after Surgical Intervention. 


\section{Otolaryngology Open Access Journal}

Yonsei Med J 53(3): 642-648.

5. Ikeda M, Nakazato H, Onoda K, Hirai R, Kida A (2006) Facial nerve paralysis caused by middle ear cholesteatoma and effects of surgical intervention. Acta Otolaryngol 126(1): 95-100
6. Mehta RP (2009) Surgical Treatment of Facial Paralysis. Clin Exp Otorhinolaryngol 2(1): 1-5.

7. Kumar SS, Thakar A (2012) Spectrum of facial paralysis in chronic suppurative otitis media. Indian J Otol 18(2): 92-94. 